\title{
Saberes, desafios e perspectivas sobre a sistematização da assistência de enfermagem
}

\author{
Knowledge, challenges and perspectives on \\ systematization of nursing assistance
}

\author{
Fernanda Batista Oliveira Santos ${ }^{1}$ (1) \\ lago Leonardo Vieira da Silva² (1) \\ Bianca Santana Dutra ${ }^{3}$ (1) \\ Júlio César Batista Santana ${ }^{4}$ \\ Fernanda Alves dos Santos Carregal'5 (D) \\ Jaqueline Almeida Guimarães Barbosa ${ }^{6}$ (i)
}

1'Autora para correspondência. Universidade Federal de Minas Gerais (Belo Horizonte). Minas Gerais, Brasil. fernandabosufmg@gmail.com

${ }^{2-4}$ Centro Universitário de Sete Lagoas (Sete Lagoas). Minas Gerais, Brasil. iagoenfermagem@gmail.com, bianca27santana@yahoo.com.br, julio.santana@terra.com.br 5-6Universidade Federal de Minas Gerais (Belo Horizonte). Minas Gerais, Brasil. fernanda.carregal@hotmail.com, jaqueline@task.com.br

\begin{abstract}
RESUMO | OBJETIVO: Compreender os saberes dos enfermeiros atuantes na unidade de clínica médica adulto de um hospital de ensino bem como os seus desafios e perspectivas sobre a Sistematização da Assistência de Enfermagem. MÉTODO: Estudo de abordagem qualitativa, utilizando-se da análise de conteúdo de nove entrevistas com enfermeiros de um hospital público de ensino de grande porte. RESULTADOS: Os enfermeiros, mesmo reconhecendo a importância da sistematização da assistência de enfermagem, apresentam um conhecimento superficial do tema; vivem desafiados pelas precárias condições de trabalho institucionais, somadas a sobrecarga de atividades, o que dificulta a implementação e continuidade do cuidado sistematizado; e, implementar e continuar o cuidado sistematizado constitui uma reafirmação do cunho científico da profissão bem como estratégia para melhora do cuidado prestado. CONCLUSÃO: A sistematização da assistência de enfermagem no cotidiano do enfermeiro perpassa por subsídios profissionais e institucionais, sendo o último o de maior impacto, o que desvela um cenário dificultador do cuidado de enfermagem que deve ser pensado e discutido para o avanço da qualidade assistencial.
\end{abstract}

DESCRITORES: Enfermagem. Processo de enfermagem. Cuidados de enfermagem. Serviço hospitalar de enfermagem.
ABSTRACT | OBJECTIVE: To understand the knowledge of nurses working in the adult medical clinic unit of a teaching hospital as well as their challenges and perspectives on Systematization of Nursing Care. METHOD: Study with a qualitative approach, using the content analysis of nine interviews with nurses from a large public teaching hospital. RESULTS: Nurses, even recognizing the importance of systematization of nursing care, have a superficial knowledge of the topic; they are challenged by the precarious institutional working conditions, added to the overload of activities, which hinders the implementation and continuity of systematic care; and to implement and to continue systematic care constitutes a reaffirmation of the scientific nature of the profession as well as a strategy for the improvement of the care provided. CONCLUSION: Systematization of nursing care in the daily lives of nurses involves professional and institutional subventions, where the latter is the one with the greatest impact, which reveals a scenario hindering nursing care that should be thought about and discussed for the advancement of care quality.

DESCRIPTORS: Nursing. Nursing process. Nursing care. Nursing service. Hospital. 


\section{Introdução}

A enfermagem vem buscando autonomizar seu escopo de ser e existir, demonstrando a transição entre o empírico e a ciência, e a essência que norteia este processo de profissionalização é o cuidado. Este se originou a partir do desejo de perpetuar a vida, conceituando-o como um valor e bem social ${ }^{1,2}$. $\mathrm{Na}$ trajetória de busca pela consolidação da enfermagem como ciência, a Sistematização da Assistência de Enfermagem (SAE), regulamentada no Brasil pela Resolução COFEN 358/2009, apresenta-se como um delineamento que confere autonomia ao profissional enfermeiro ${ }^{3}$. A assistência de enfermagem deve ser sistematizada implantando-se o processo de enfermagem, porém é necessário que este seja guiado por uma teoria de enfermagem, arcabouço teórico filosófico, com o intuito de possibilitar a operacionalização dos resultados assistenciais, compondo uma linguagem própria que apoia a consolidação da enfermagem como ciência na área da saúde ${ }^{4}$.

Na esfera do cuidado, o hospital constitui-se historicamente como um cenário fundamental. O cuidado pode e deve ser exercido em todos os âmbitos dos serviços de saúde e o cuidado hospitalar compõe uma parcela importante no reestabelecimento da saúde da clientela. A hospitalização traz alterações profundas na vida do paciente e de sua família por ser uma vivência especialmente estranha, desconhecida e impactante para o paciente ${ }^{5}$. A equipe de enfermagem possui um papel fundamental neste ponto da assistência, de modo que as ações deverão ser individualizadas ${ }^{6}$.

O enfermeiro que trabalha no ambiente hospitalar desempenha funções que têm o objetivo de recuperar a saúde dos pacientes, quando possível ou minimizar seu sofrimento e, a sua prática assistencial deve ser pautada em um modelo próprio, que organize a assistência em saúde permitindo a realização de uma prática desenvolvida com base nas singularidades de cada paciente ${ }^{7,8}$.
Mesmo sabendo dessas premissas e de que a sistematização da assistência de enfermagem é uma condição obrigatória prevista pelo Conselho Federal de Enfermagem, o que se observa é a necessidade dos serviços de saúde implementar o processo de enfermagem, o que viabiliza a organização da assistência ao paciente. Pode-se pressupor que isto ainda aconteça pelo desconhecimento da sistematização por parte dos profissionais que compõem a equipe de enfermagem. Assim, esta pesquisa tomou como questão norteadora "O enfermeiro possui saberes acerca da SAE para aplicá-la em unidade de clínica médica adulto de um hospital de ensino? Quais seriam os seus desafios e perspectivas frente a esta execução?"

A importância desse trabalho se deve à primordialidade da execução da SAE em todos os ambientes conforme definido pela legislação supracitada. Além disso, essa pesquisa visa colaborar com o avanço da assistência de enfermagem em relação às suas técnicas assistenciais, contribuindo para o aperfeiçoamento do cuidado, segurança do paciente e reiteração do cunho científico da profissão ${ }^{3}$. Nesse sentido, a identificação do nível de conhecimento da equipe de enfermagem sobre a SAE é de extrema relevância, a fim de propor soluções que contribuam para a sua viabilização, com o propósito de aprimorar esta metodologia de trabalho, aumentando a qualidade da assistência e valorizando a prática do profissional ${ }^{9}$.

A valorização da enfermagem depende da atuação profissional de cada um e de seu conhecimento técnico-científico. Estudo aponta que existem lacunas no conhecimento sobre $S A E$, sendo necessário aprimorar o ensino, estimular a qualificação profissional e treinamento da equipe para utilização dos instrumentos relacionados ao Processo de enfermagem. Com a SAE o enfermeiro pode então conquistar status profissional e proporcionar um atendimento de qualidade ${ }^{10}$. Sendo assim, esse estudo teve como objetivo compreender os saberes dos enfermeiros atuantes na unidade de clínica médica adulto de um hospital de ensino bem como os seus desafios e perspectivas sobre a Sistematização da Assistência de Enfermagem. 


\section{Método}

Trata-se de um estudo de abordagem qualitativa, pois o interesse é a busca do significado das vivências dos enfermeiros sobre a implantação da SAE, buscando uma análise aprofundada dos achados. Contudo, os significados que as vivências ganham, passam também a ser partilhados culturalmente e assim organizam o grupo social em torno destas representações e simbolismos ${ }^{11}$.

O local de realização do estudo foi uma Unidade de Clínica Médica Adulto do Hospital Risoleta Tolentino Neves, situado na região metropolitana de Belo Horizonte. O perfil de pacientes é constituído por ênfase nos cuidados paliativos, ao idoso frágil, cuidado pós acidente vascular encefálico e cuidado semi-intensivo. A unidade conta com 29 enfermeiros. Foram realizados agendamentos em todos os turnos para a realização das entrevistas gravadas. Estas ocorreram durante o turno de trabalho dos participantes e foi reservada uma sala específica para a coleta. O período de realização da coleta de dados do estudo ocorreu nos meses de janeiro e fevereiro de 2019.

Os participantes da pesquisa foram nove enfermeiros. Os critérios de inclusão foram: atuar na Clínica Médica do cenário do estudo. Como critério de exclusão determinou-se ter menos de três meses de atuação no setor, estar em período de férias e licença saúde. A coleta de dados ocorreu por meio de entrevistas utilizando um roteiro semiestruturado. Adotou-se a saturação amostral considerando a repetição dos conteúdos de falas. Após a coleta foi feita a transcrição e codificação denominando os enfermeiros pela letra E seguida de um número para garantir o tratamento ético dos dados.

Posteriormente realizou-se a análise de conteúdo, segundo Laurence Bardin ${ }^{12}$, em três polos cronológicos: pré-análise, momento no qual ocorreu a organização propriamente dita; exploração do material que consistiu na codificação dos resultados; tratamento dos resultados, a inferência e a interpretação, momento no qual os resultados foram tratados de maneira a serem significativos e válidos. A categorização analítica foi delineada após a análise, considerando as unidades de conteúdo mais expressivas entre os achados ${ }^{12}$.
Este estudo seguiu os critérios éticos estabelecidos pela pesquisa com seres humanos e recebeu parecer favorável do Núcleo de ensino, pesquisa e extensão do hospital, seguido da submissão do projeto ao Comitê de ética em pesquisa, iniciando-se a coleta após a autorização deste e da assinatura do termo de consentimento livre e esclarecido pelos participantes.

\section{Resultados e discussão}

Colaboraram neste estudo um grupo de nove enfermeiros, sendo destes oito mulheres e um homem. Percebeu-se que destes, a profissional com menor tempo de formação possui dois anos de formada, e a com maior tempo, possui 18 anos de formada. Sete dos nove entrevistados possuem pós-graduação latu senso e apenas um possui strictu senso. Quanto ao tempo de atuação em clínica médica, a com menor tempo de atuação possui três meses e a com maior tempo dezesseis anos. No que tange à instituição de formação, nota-se que oito são egressos de instituições localizadas no estado de Minas Gerais e, apenas um profissional é egresso de uma instituição paulista, sendo a maioria destas instituições de iniciativa privada.

Durante a análise das entrevistas percebeu-se que apenas um dos entrevistados não havia tido uma disciplina específica relativa ao conteúdo de SAE durante a graduação, sendo este o profissional de maior tempo de formação. Inferiu-se que esse profissional se graduou no ano de 2000 , sendo a SAE regulamentada pela primeira vez no ano de 2002 pela Resolução do COFEN 272 de 2002.

A partir da análise das entrevistas foi possível delinear as seguintes categorias temáticas: "Sistematização da Assistência de Enfermagem: do que estamos falando?"; "A SAE como instrumento metodológico para a prática da enfermagem"; "Desvelando os desafios para a execução da SAE na práxis da enfermagem atuante em clínica médica"; "A perspectiva do enfermeiro acerca da implantação da Sistematização da Assistência de Enfermagem". 


\section{Conhecimento da Sistematização da Assistência de Enfermagem}

Esta categoria surgiu das respostas dos enfermeiros quando questionados sobre o conceito de SAE. Após análise das falas apresentadas pelos profissionais, pode-se inferir que os enfermeiros reconhecem a SAE como um instrumento para organização do serviço de enfermagem e da prestação de cuidados, conforme evidenciado pelas falas que seguem:

Sae é para sistematizar a assistência de enfermagem, né?!, e tem as etapas, né ?! (E1).

Serve para organização do setor né? Para direcionar o trabalho da equipe de enfermagem. (E2)

É a organização do serviço de enfermagem para o cuidado de um todo do paciente. (E3)

Processo lógico que orienta as futuras ações no plano de cuidado com o paciente. (E4)

Após a análise destas falas, infere-se que os participantes deste estudo, mesmo reconhecendo importância da utilização da SAE, apresentam um conhecimento básico deste instrumento. Tais achados corroboram com uma pesquisa realizada por Silva e colaboradores $^{13}$ que contou com a participação de oito enfermeiros. Segundo este estudo, quanto aos enfermeiros, seis desses, ao responderem sobre o que compreendem em relação ao conceito de $S A E$ e $P E$, demonstraram superficialidade nas definições. Esta característica pode ser percebida quando se observa que em nenhum momento da entrevista os profissionais citaram as fases do PE bem como a relação entre este e as teorias de enfermagem.

Um fator que pode estar relacionado com a superficialidade do conhecimento sobre SAE, é a fragmentação dos conteúdos, não inter-relacionando com as teorias de enfermagem. Um estudo realizado com trinta e dois acadêmicos de enfermagem em uma instituição estadual observou que os conteúdos relacionados à SAE foram apresentados de forma fragmentada, provocando alguns conflitos nos acadêmicos, que só compreenderam a razão deste conhecimento a partir da metade do curso $^{14}$.
Em um estudo realizado com sessenta e seis acadêmicos do último ano do curso de enfermagem de uma instituição privada amazonense, apresentou alguns relatos que comprovam que o ensino da $S A E$ ainda está aquém do desejado, devido a deficiências no ensino teórico-prático desta metodologia ${ }^{15}$.

Outra importância apresentada para a SAE está relacionada com a integralidade do cuidado de enfermagem e avaliação holística do paciente:

É o cuidado que o enfermeiro tem em relação ao paciente [...] você avalia o paciente e tem como você prescrever e estar mais próximo do paciente para os cuidados [...] (E5)

É uma forma de uniformizar e dar assistência unificada a cada paciente de acordo com a sua necessidade. (E6)

Por meio da SAE é possível realizar o plano de cuidado individualizado, prescrição de enfermagem para cada paciente, o enfermeiro aproxima do paciente, aumentando o vínculo (E9)

A SAE é um instrumento de gestão do cuidado facilitador do cuidado realizado pela equipe de enfermagem, visando uma assistência de qualidade. Este instrumento pode ajudar na adaptação do paciente ao tratamento e dispensar a ele um cuidado individualizado ${ }^{16}$. Cabe salientar que a utilização da SAE, possibilita ao enfermeiro o desenvolvimento de um cuidado holístico, atendendo às necessidades e especificidades do paciente.

Na fala acima (E9), outro fator importante pode ser percebido: a criação do vínculo entre o profissional e o paciente. $O$ vínculo entre o enfermeiro e o usuário possui influência direta no sucesso de um determinado tratamento, consistindo em uma estratégia de promoção da integralidade e singularidade do cuidado em saúde ${ }^{17}$.

\section{A SAE como instrumento de gestão para a práti- ca da enfermagem: implicações vivenciadas no cotidiano assistencial perante a implantação do Processo de Enfermagem}

Esta categoria advém de falas comuns entre os profissionais entrevistados que reconhecem a realização da SAE como ferramenta de reconhecimento científico 
da profissão perante as demais profissões da área da saúde. A SAE visa a organização e planejamento das ações que são realizadas pela equipe de enfermagem durante o período de internação dos pacientes ${ }^{18}$. Ao analisar as falas, pode-se observar este reconhecimento por parte dos entrevistados:

A SAE ela é a modulação científica da prática da enfermagem, uma forma de trazer o fazer da enfermagem de maneira científica não baseada em empirismo e sim correlacionada em estudos científicos, em práticas direcionadas no ato da enfermagem. (E7)

Para mim, a SAE é um dos mais importantes instrumentos para a sistematização, padronização e viabilização da qualidade nos processos e rotinas desempenhadas pelo enfermeiro, na sua prática diária. (E8)

Eu acho que a partir dela a gente consegue ver melhor o trabalho do enfermeiro, a gente consegue direcionar mais o cuidado [...]. (E9)

Os resultados obtidos através das entrevistas corroboram com os resultados encontrados em uma pesquisa realizada em uma maternidade que contou com a participação de treze enfermeiros. Os enfermeiros reconhecem que a aplicação do processo de enfermagem permite a identificação de necessidades, promove o planejamento e a organização do cuidado, bem como facilita a prática assistencial do enfermeiro ${ }^{19}$. Tal reconhecimento pode ser percebido nas falas a seguir:

É importante porque traz uma melhoria, um cuidado mais holístico, um cuidado melhor para o paciente, porque não fica uma coisa muito desconexa, né um cuidado do outro, serve pra juntar tudo, orientar melhor a equipe né. (E3)

Como eu trabalho a noite às vezes a gente não conhece o paciente, então a sistematização já dá um direcionamento maior do que é que aconteceu durante o dia, a gente consegue conhecer mais o paciente. (E9)
A sistematização da assistência de enfermagem, enquanto processo organizacional é capaz de oferecer subsídios para o desenvolvimento de métodos/ metodologias interdisciplinares e humanizadas de cuidado $^{20}$. Tomando os dados supracitados podemos observar que os profissionais de enfermagem reconhecem a SAE como instrumento de gestão que orienta a práxis da enfermagem e possibilitam o desenvolvimento de um plano de ação individualizado de acordo com as necessidades de cada paciente. Tal metodologia promove também um reconhecimento da enfermagem como ciência do cuidado, promovendo a transposição entre o cuidado empírico e científico.

\section{Desvelando os desafios para a execução da SAE na práxis da enfermagem atuante em clínica médica}

Esta categoria surgiu da análise que apresentava as barreiras cotidianas que impedem e/ou dificultam a execução de todas as etapas constituintes da SAE. Apesar de ser obrigatória, a SAE não é realizada de forma adequada pelo enfermeiro devido à sobrecarga de trabalho imposta a esse profissional, que acaba se detendo em atividades burocráticas e administrativas, que também fazem parte de suas atribuições profissionais ${ }^{21}$. Estes fatores também foram citados, conforme pode-se demonstrar nas falas abaixo:

A série de atribuição do enfermeiro ser incompatível com a assistência direta do paciente...eu de manhã ficando com 24 pacientes, o enfermeiro da tarde sendo responsável por 24 e na noite com 24 não há possibilidade de prestar um trabalho sistematizado, uma assistência sistematizada a um paciente. (E7)

O excesso de pacientes e a complexidade [...] às vezes a equipe não está dividida de acordo com a complexidade [...]. (E6)

Nas falas supracitadas, pode-se perceber que um dos fatores dificultadores da execução da SAE é o dimensionamento dos profissionais de enfermagem. 
Esta ferramenta de gestão possibilita uma distribuição de profissionais de enfermagem de acordo com o grau de complexidade dos cuidados exigidos pelos pacientes. Desta forma, possibilita uma atenção à saúde pautada na equidade. $O$ dimensionamento constitui-se em recurso indispensável para o provimento do pessoal de enfermagem, em uma abordagem quantitativa e qualitativa, considerada a base para a organização da equipe necessária para o atendimento das necessidades dos pacientes, em consonância com o grau de dependência por eles apresentados ${ }^{22}$.

Outro fator citado como desafio para a aplicação da SAE foi a ausência de capacitações para os profissionais e alta rotatividade, além de ausência de equipamentos (tecnologia dura). Essas evidências encontram-se logo abaixo:

Alta rotatividade de profissionais no setor. Falta de treinamento para os profissionais do plantão noturno. Pouca motivação na implantação e aplicação da SAE. (E4)

Temos os enfermeiros, bastante enfermeiro, muitas vezes não temos os equipamentos, há um número menor de computadores. (E3)

Eu acho que principalmente no sistema, utilizar um sistema que seja coerente com as nossas necessidades. (E9)

Temos o prontuário informatizado né, então assim, já tivemos acesso a prescrição de enfermagem, mas são milhares de itens, mas isso precisa ser organizado melhor, sabe [...]. (E2)

Pode-se realizar uma correlação importante entre a aplicação da SAE com as Tecnologias de Cuidado. As tecnologias referidas podem ser classificadas em duras, leve-duras e leves, e estas se inter-relacionam à medida que surge a necessidade de implementá-las na assistência ${ }^{23}$. A principal pontuação dos profissionais participantes deste estudo refere-se ao número insuficiente de computadores, ou seja, tecnologia dura, e também à ausência de reunião/capacitações que se enquadram nas tecnologias leves. A utilização destas tecnologias no processo de enfermagem faz-se necessária e indispensável, visto que o mesmo é a dinâmica das ações sistematizadas e inter-relacionadas que visa à assistência ao ser humano'.
A ausência de treinamento também foi apontada como dificultador da execução da SAE:

Acredito que deve ser realizada uma ampla discussão entre os gestores e coordenadores a respeito da melhor forma de dar início à aplicação da SAE na instituição, assim como um treinamento aos enfermeiros de forma a esclarecê-los e conscientizálos sobre a relevância da SAE nas rotinas de trabalho, assim como suas contribuições. (E8)

Um estudo aponta resultados que corroboram com os encontrados nesta pesquisa, ao evidenciar que o número reduzido de enfermeiros, a ausência de educação permanente, além do desconhecimento e inabilidade na operacionalização das etapas do PE foram fatores elencados como dificultadores da execução da SAE, atrelando-se a isto também, o excesso de atividades burocráticas ${ }^{13}$.

Para que se possa ofertar um cuidado seguro e uma aplicação completa da SAE, outro fator essencial é a capacitação profissional e educação permanente. As ações de educação oferecidas pelas instituições de saúde objetivam mudanças positivas na postura profissional e melhoria da qualidade da assistência prestada $^{19}$. A Educação permanente se caracteriza pela problematização dos processos de trabalho em saúde com a participação de profissionais para corresponder às necessidades de saúde da população. Na área da saúde há uma necessidade de aprimorar o ensino nas instituições de formação técnica e superior, para uma proposta de educação crítica e reflexiva contribuindo para a construção permanente do SUS que todos almejam ${ }^{10}$.

Uma pesquisa realizada em uma universidade do Rio Grande do Norte desenvolveu uma plataforma digital para capacitação dos profissionais de enfermagem quanto a SAE. O objeto virtual de aprendizagem possibilita o desenvolvimento de um recurso a ser usado tanto na educação presencial quanto a distância, pois permite o estabelecimento de situações de efetiva aprendizagem em uma ou várias áreas do conhecimento ${ }^{13}$. Sendo assim, é necessário que além do interesse dos profissionais em se capacitar, o próprio gestor do serviço de saúde estimule a capacitação e especialização destes profissionais, visando assim uma melhoria na assistência prestada ${ }^{16}$. 


\section{A perspectiva do enfermeiro acerca da implantação da Sistematização da Assistência de Enfermagem}

A SAE constitui-se em um instrumento de gestão que visa organizar a prática da equipe da enfermagem, permitindo a práxis. Com base na Resolução COFEN 358/2009, considerando a organização do trabalho da equipe de enfermagem, e as falas dos entrevistados, é possível delinear as perspectivas do enfermeiro com a aplicação da SAE no seu cuidado3:

A SAE bem organizada consegue padronizar os processos de trabalho. Evitando a divergência entre profissionais. (E4)

Visualizo um cenário em que as práticas serão realizadas de forma mais completa, sistemática e alinhada entre os enfermeiros, o que irá refletir positivamente na equipe como um todo e principalmente nos cuidados prestados aos pacientes. (E7)

Realmente, melhoria da assistência, da qualidade da assistência que a gente visa né. (E2)

Acho que ia melhorar o atendimento de enfermagem né, valorizar o enfermeiro, porque as outras profissões conseguem visualizar melhor o nosso trabalho. (E9)

A implantação desse aparato de gestão é fundamental no reconhecimento do real papel da enfermagem $^{15}$. A SAE possibilita uma maior visibilidade das ações do enfermeiro frente aos demais profissionais de saúde e comunidade. Implementar este instrumento reafirma a cunho científico da profissão e a adoção da prática baseada em evidências como estratégia para melhora do cuidado prestado.

Os achados desta categoria corroboram com um estudo que abordou a temática da SAE como instrumento para autonomia do enfermeiro. A visibilidade do trabalho do enfermeiro em relação à equipe e à comunidade está interligada com a atuação dos enfermeiros ao sair da invisibilidade ${ }^{24}$.

Outra ação que visa aumentar a visibilidade deste profissional é a campanha mundial Nursing Now. Este movimento pela valorização da enfermagem tem por metas: aumentar o investimento na melhoria da educação, do desenvolvimento profissional, da regulação e das condições de trabalho para enfermeiros; aumentar a influência destes nas políticas nacionais e internacionais; aumentar o número de enfermeiros em posições de liderança com mais oportunidades para desenvolvimento em todos os níveis de liderança; aumentar as evidências que apoiem as políticas; trabalhar para que os enfermeiros atuem integralmente; e aumentar e melhorar a disseminação de práticas de enfermagem efetivas e inovadoras ${ }^{25}$.

A aplicação da SAE eleva o nível de assistência ofertado ao paciente, sendo de grande importância uma vez que auxilia a enfermagem no desenvolvimento de suas atividades, orientando a realização de um cuidado individualizado, beneficiando assim o cliente/paciente ${ }^{16}$. Este benefício ao paciente foi apontado como perspectivas pelos participantes deste estudo, conforme as falas abaixo:

A questão do cuidado vai melhorar, vai ser mais objetivo[...] seria muito melhor pro paciente. O ganho será muito melhor. (E5)

Só melhorias, eu acho que essa melhoria não só da gente como profissional, eu acho que quem ganha mais

é o paciente né, na questão mesmo do cuidado. (E3)

Um estudo realizado com acadêmicos de enfermagem apontou que ao se utilizar a SAE consegue-se promover aos pacientes os cuidados qualificados em menor tempo, agilizando dessa maneira o atendimento ao paciente ${ }^{15}$. Destarte pode-se inferir que sistematizar a assistência possui inúmeros benefícios, tanto aos profissionais quanto aos pacientes, favorece a comunicação, fortalece o vínculo profissional-paciente e contribui para a visibilidade profissional.

\section{Considerações finais}

O estudo mostra o interesse dos enfermeiros em sistematizar a assistência de enfermagem, uma vez que reconhecem os benefícios para a autonomia da profissão e para o cuidado do paciente. No entanto, são confrontados com uma série de desafios/dificuldades conceituais, operacionais e institucionais, esta 
última a principal, que não permite que a sistematização do cuidado avance de forma plena. Continuase acreditando que a visibilidade das ações do enfermeiro perante os demais profissionais da área da saúde mora nas ações sistematizadas e que é por meio dela que será possível galgar mais status e autonomia no campo profissional.

O estudo possui como limitação o fato de não permitir generalizações para outros cenários brasileiros, uma vez que os dados referem-se a apenas uma instituição hospitalar e este guarda as singularidades culturais e políticas de seu estado. No entanto, tal limitação faz um chamamento para a proposição de novas pesquisas em outras regiões do país, em instituições hospitalares e não-hospitalares, permitindo uma melhor e mais ampliada caracterização dos saberes, desafios e perspectivas da SAE pelos enfermeiros brasileiros.

\section{Contribuições dos autores}

Silva ILV e Dutra BS participaram da concepção, delineamento, busca e análise dos dados da pesquisa, interpretação dos resultados, redação do artigo científico. Carregal FAS, Santana JCB e Barbosa JAG participaram da análise dos dados e redação do artigo científico. Santos FBO participou da concepção, delineamento, análise dos dados da pesquisa, interpretação dos resultados e redação.

\section{Conflitos de interesses}

Nenhum conflito financeiro, legal ou político envolvendo terceiros (governo, empresas e fundações privadas, etc.) foi declarado para nenhum aspecto do trabalho submetido (incluindo mas não limitando-se a subvenções e financiamentos, participação em conselho consultivo, desenho de estudo, preparação de manuscrito, análise estatística, etc.).

\section{Referências}

1. Sousa SM, Bernardino E, Crozeta K, Peres AM, Lacerda MR. Cuidado integral: desafio na atuação do enfermeiro. Rev Bras Enferm. 2017;70(3):504-510. doi: 10.1590/0034-7167-2016-0380

2. Magalhães ALP, Erdmann AL, Sousa FGM, Lanzoni GMM, Silva EL, Mello ALSF. Significados do cuidado de enfermagem ao paciente em morte encefálica potencial doador. Rev Gaúcha Enferm. 2018;39:e2017-0274. doi: 10.1590/1983-1447.2018.2017$\underline{0274}$
3. COFEN. Resolução COFEN n 358/2009. Brasília; 2009.

4. Tannure MC, Pinheiro AM. SAE: Sistematização da Assistência de Enfermagem, guia prático. 2.ed. Rio de Janeiro: Guanabara Koogan; 2019.

5. Santos MFG, Almeida IS, Reis NSP, Leite DC, Gomes HF, Costa AJ. A Percepção da Hospitalização pelos Adolescentes: Contribuições para o Cuidado de Enfermagem. Rev Fund Care Online. 2018;10(3):663-668. doi: 10.9789/2175-5361.2018.v10i3.663-668

6. Silva AT, Camelo SHH, Terra FS, Dázio EMR, Sanches RS, Resck ZMR. Segurança do paciente e a atuação do enfermeiro em hospital. Rev enferm UFPE on line. 2018;12(6):1532-8. doi: 10.5205/1981-8963-v12i6a234593p1532-1538-2018

7. Maia SMS, Souza SR, Sória DAC, Costa TB. A Resiliência do Enfermeiro de Clínica Médica e Cirúrgica em seu Cuidado Cotidiano. Rev enferm UFPE on line. 2017;11(6):3093-9. doi: 10.5205/reuol.11064-98681-4-ED.1108201714

8. Rocha EN, Lucena AF. Projeto Terapêutico Singular e Processo de Enfermagem em uma perspectiva de cuidado interdisciplinar. Rev Gaúcha Enferm. 2018;39:e2017-0057. doi: 10.1590/19831447.2018.2017-0057

9. Mola R, Dias ML, Costa JF, Fernandes FECV, Lira GG. Conhecimento dos profissionais de enfermagem sobre a sistematização da assistência de enfermagem. Rev Fun Care Online. 2019;11(4):887-893. doi: 10.9789/2175-5361.2019. v11i4.887-893

10. Mola R, Dias ML, Costa JF, Fernandes FECV, Lira GG. The nursing professionals' knowledge with regards to the nursing care systematization. Rev Fun Care Online. 2019;11(4):887-893. doi: 10.9789/2175-5361.2019.v11i4.887-893

11. Yin RK. Pesquisa qualitativa do início ao fim. Porto Alegre: Penso; 2016.

12. Bardin L. Análise de Conteúdo. São Paulo: Edições 70; 2016.

13. Silva RS, Almeida ARLP, Oliveira FA, Oliveira AS, Sampaio MRFB, Paixão GPN. Sistematização da Assistência de Enfermagem na perspectiva da equipe. Enferm Foco. 2016;7(2):32-36.

14. Silva JP, Garanhani ML, Peres AM. Sistematização da Assistência de Enfermagem na graduação: um olhar sob o Pensamento Complexo. Rev Latino-Am Enfermagem. 2015;23(1):59-66. doi: 10.1590/0104-1169.0096.2525

15. Meneses ARC, Goiabeira YNLA, Menezes EG, Lima ABS, Jardim MJA, Luiz Neto M. Dificuldades dos acadêmicos de enfermagem na aplicabilidade da sistematização da assistência de enfermagem. Rev Fun Care Online. 2019;11(1):181-185. doi: 10.9789/2175-5361.2019.v11i1.181-185 
16. Silva AR, Forte ECN, Padilha MI, Vieira AN. Contributions of the Nursing Care Systematization to the Chronic Kidney Patient: An Integrative Review. Rev Fund Care Online. 2019;11(3):700-706. doi: 10.9789/2175-5361.2019.v11i3.700-706

17. Santos RCA, Miranda FAN. Importância do vínculo entre profissional-usuário na estratégia de saúde da família. Rev Enferm UFSM. 2016;6(3):350-359. doi: 10.5902/2179769217313

18. Neco KKS, Costa RA, Feijão AR. Sistematização da assistência de enfermagem em instituições de saúde no Brasil: revisão integrativa. Rev enferm UFPE on line. 2015;9(1):193-200. doi: 10.5205/reuol.6817-60679-1-ED.0901201527

19. Dotto Jl, Backes DS, Dalcin CB, Lunardi Filho WD, Siqueira $\mathrm{HCH}$, Zamberlan C. Sistematização da assistência de enfermagem: ordem, desordem ou (re)organização? Rev enferm UFPE on line. 2017;11(10):3821-9. doi: 10.5205/reuol.12834-30982-1SM.1110201716

20. Gutiérrez MGR, Morais SCRV. Sistematização da Assistência de Enfermagem e a formação da identidade profissional. Rev Bras Enferm. 2017;70(2):455-60. doi: 10.1590/0034-7167-2016-0515

21. Tavares FMM, Tavares WS. Elaboração do instrumento de sistematização da assistência de enfermagem: relato de experiência. Revista de Enfermagem do Centro-Oeste Mineiro. 2018. doi: $10.19175 /$ recom.v7i0.2015

22. Vandresen L, Pires DEP, Lorenzetti J, Andrade SR. Classificação de pacientes e dimensionamento de profissionais de enfermagem: contribuições de uma tecnologia de gestão. Rev Gaúcha Enferm. 2018;39:e2017-0107. doi: 10.1590/19831447.2018.2017-0107

23. Merhy EE. Saúde: a cartografia do trabalho vivo. São Paulo: Hucitec; 2002.

24. Bonfada MS, Pinno C, Camponogara S. Potencialidades e limites da autonomia do enfermeiro em ambiente hospitalar. Rev enferm UFPE on line. 2018;12(8):2235-46. doi: 10.5205/1981-8963-v12i8a234915p2235-2246-2018

25. Cassiani SHB, Lira Neto JCG. Perspectivas da Enfermagem e a Campanha Nursing Now. Rev Bras Enferm. 2018;71(5):2351-2352. doi: 10.1590/0034-7167.2018710501 